\title{
Priorización de despachos en empresas de manufactura usando QFD difuso*
}

\author{
Juan Carlos Osorio Gómez** \\ Mayra Alejandra Peña Vega ${ }^{* * *}$ \\ Deysi Yulieth Arias Giraldo ${ }^{* * *}$
}

\author{
Recibido: 18/03/2016 - Aceptado: 26/02/2018 \\ https://doi.org/10.22395/rium.v17n33a9
}

\begin{abstract}
Resumen
Las restricciones de capacidad instalada y de recursos de distribución a las que normalmente se encuentran sometidas las medianas y grandes empresas manufactureras, hacen que surja la necesidad de establecer métodos que sirvan como herramienta para tomar decisiones en cuanto a la priorización de cada una de sus bodegas al momento de abastecerlas. La importancia que el centro de distribución asigna a cada una de estas, se encuentra estrechamente relacionada con las condiciones y particularidades que las caracterizan, pudiendo ser estos factores de tipo cualitativo, cuantitativo o una mezcla entre los dos. Este artículo presenta un método multicriterio QFD difuso el cual, además de utilizar variables cuantitativas para establecer el orden de despacho de producto terminado, tiene en cuenta variables cualitativas, resultado de las opiniones que los expertos pueden ofrecer acerca de las situaciones particulares de cada bodega mediante variables lingüísticas que, finalmente, se cuantifican a través de números difusos triangulares.
\end{abstract}

Palabras clave: despliegue de la función de la calidad difuso; toma de decisiones multicriterio; priorización de despachos; QFD; lógica difusa.

* $\quad$ Proyecto derivado de la investigación en el marco del trabajo de grado de pregrado en Ingeniería Industrial realizado por las autoras Peña y Arias bajo la dirección del autor Osorio. Proyecto titulado Formulación de un modelo para priorización de despacho de producto terminado en empresas manufactureras usando QFD fuzzy. El proyecto fue financiado por los autores con recursos propios y se ejecutó entre 2015 y 2016.

** Doctor en Ingeniería de la Universidad del Valle. Grupo de investigación Logística y Producción. Calle 13 N. ${ }^{\circ}$ 100-00, Cali, Colombia. Teléfono: (+57) 23212100 ext. 7545. Correo electrónico: juan.osorio@correounivalle. edu.co. Orcid: http://orcid.org/0000-0001-5625-5609

*** Ingeniera Industrial de la Universidad del Valle. Carrera 94 N. 34-55, apto. 302 E Balcones del Lili, Cali, Colombia. Teléfono: (+57) 3113167993. Correo electrónico: mayralejandrapv@outlook.com. Orcid: https://orcid. org/0000-0003-2759-8578

***** Magíster en Administración de la Universidad del Valle. Km. 4 vía a Potrerito, Condominio campestre Haciendas de Potrerito, Hacienda Garzonero 17. Teléfonos: (+57) 3107409912 - 3116916673 . Correo electrónico: yulietharias@hotmail.com. Orcid: https://orcid.org/0000-0001-5344-8122 


\title{
Prioritization of Deliveries in Manufacturing Companies Using Fuzzy QFD
}

\begin{abstract}
The restrictions of installed capacity and distribution resources to which medium and large manufacturing companies are normally subject, make it necessary to establish methods that serve as a tool to make decisions regarding the prioritization of each of their warehouses at the time of supplying them. the importance that the distribution center assigns to each of these, is closely related to the conditions and particularities that characterize them, being these factors of a qualitative, quantitative or a mixture between the two. This article presents a fuzzy QFD multi-criteria method which, in addition to using quantitative variables to establish the order of dispatch of the finished product, considers qualitative variables, the result of the opinions that experts can offer about the particular situations of each winery by means of linguistic variables that are finally quantified through fuzzy triangular numbers.
\end{abstract}

Keywords: fuzzy quality function deployment; multi-criteria decision making; dispatch prioritization; QFD; fuzzy logic.

\section{Priorização de despachos em empresas de manufatura usando QFD difuso}

\section{Resumo}

As restrições de capacidade instalada e de recursos de distribuição às quais as médias e grandes empresas manufatureiras normalmente se encontram submetidas fazem com que surja a necessidade de estabelecer métodos que sirvam como ferramenta para tomar decisões com respeito à priorização de cada um de seus estoques na hora de abastecê-los. A importância que o centro de distribuição designa a cada um deles está estreitamente relacionada às condições e particularidades que os caracterizam. Tais fatores podem ser de tipo qualitativo, quantitativo ou uma mistura dos dois. Este artigo apresenta um método multicritério QFD difuso que, além de utilizar variáveis quantitativas para estabelecer a ordem de despacho do produto terminado, leva em consideração variáveis qualitativas, resultados das opiniões que os especialistas podem oferecer sobre as situações particulares de cada estoque por meio de variáveis linguísticas que, finalmente, são quantificadas por meio de números difusos triangulares.

Palavras-chave: desdobramento da função da qualidade difuso; tomada de decisões multicritério; priorização de despachos; QFD; lógica difusa. 


\section{INTRODUCCIÓN}

Las medianas y grandes empresas, especialmente las manufactureras y comercializadoras de productos de consumo masivo, generalmente cuentan con centros de despacho y bodegas a abastecer, las cuales se ubican en distintas zonas geográficas. Esto implica una serie de decisiones afectadas por múltiples criterios, como disponibilidad de recursos, ubicación geográfica, vida útil del producto, demanda del producto, políticas administrativas, planes estratégicos corporativos, políticas de distribución, entre otras.

Según [1], ha existido en la literatura gran cantidad de investigaciones que abordan la necesidad de atender a los clientes ágilmente, con un transporte eficiente y oportuno, sin embargo, también afirman que no se han encontrado trabajos donde se hayan definido, de manera clara, indicadores que permitan planificar los despachos de la organización, apuntando a aquellas variables clave para el servicio al cliente y la gestión efectiva de los recursos de los que dispone una empresa, de manera que la decisión acerca de los despachos involucre múltiples criterios. En ese sentido, ellos proponen una metodología sustentada en el proceso analítico jerárquico, el cual se puede considerar como un importante antecedente para este trabajo. Sin embargo, aquí se plantea utilizar el QFD difuso como herramienta para involucrar los diferentes criterios (cuantitativos y cualitativos) a considerar en el proceso de toma de decisión respecto al despacho desde el centro de distribución a las múltiples bodegas.

Si se conocen, para cada caso específico, los requisitos que las bodegas consideran importantes para el buen desarrollo del proceso de despacho de producto terminado, la herramienta QFD permitirá relacionar dichos requisitos con los aspectos técnicos que los deben satisfacer mediante la primera matriz de la metodología conocida como casa de la calidad, que arroja como resultado un ranking de los despachos. La incorporación de la lógica difusa permitirá considerar en el modelo QFD la ambigüedad que existe en cuanto a la importancia asignada a cada uno de los requisitos y criterios. A continuación, se presentan algunos elementos teóricos importantes a considerar sobre la herramienta mencionada.

\section{La función de despliegue de la calidad (quality function deployment, QFD)}

Aunque inicialmente fue concebida como herramienta para el diseño y desarrollo de productos, ha trascendido estas aplicaciones y hoy por hoy puede considerarse como una importante herramienta en el ámbito de los problemas de decisión multicriterio.

Según [2] se pueden mencionar los siguientes campos de aplicación del QFD: desarrollo de productos, administración de la calidad, análisis de las necesidades del cliente, diseño de productos, planificación del producto y del proceso, planificación general, planificación estratégica, planificación de procesos de negocios, de calidad de 
servicios, ingeniería concurrente, ingeniería de calidad e ingeniería simultánea, toma de decisiones, administración, trabajo en equipo, programación de actividades, costeo y otros. Dos trabajos importantes para ampliar la información sobre el QFD son [2] y [3].

\section{Lógica difusa}

La modelación difusa permite la definición de métricas con las que se puede acceder a los factores intangibles y tratar con medidas heterogéneas. Además, lo que se busca a través de la matemática difusa es describir y formalizar la realidad empleando modelos flexibles que interpreten las leyes que rigen el comportamiento humano y las relaciones entre los hombres [4].

La lógica difusa permite expresar matemáticamente los valores intermedios a los que puede acudir un evaluador de determinada "situación" o "problema" de tipo cualitativo, en el cual no se sentiría satisfecho al calificar con solo dos valores, falso (0) o verdadero (1), sabiendo que es un evento que no se puede definir como totalmente verdadero o totalmente falso, sino que tiene un grado de verdad o falsedad, que puede variar de 0 a 1 . En esencia, con esta lógica se amplían las opciones frente a una situación, pues en lugar de los extremos 0 y 1 , se tiene el intervalo entre ellos para emitir un juicio. Los conjuntos difusos se manejan a partir de números difusos, los cuales pueden ser representados por funciones triangulares o trapezoidales [5].

\section{Aplicaciones de la metodología QFD difusa}

Si bien, no se ha encontrado en la literatura una aplicación de la metodología QFD difusa para la priorización de los despachos, se pueden encontrar aplicaciones recientes de QFD difuso en la toma de decisiones multicriterio en [6-12] y aplicaciones locales y específicas de la metodología se pueden encontrar en [13-15].

\section{MATERIALES Y MÉTODOS: MODELO QFD-DIFUSO PARA PRIORIZACIÓN DE DESPACHO DE PRODUCTO TERMINADO}

La metodología que se presenta a continuación está soportada fundamentalmente en el trabajo de [7]. Para el desarrollo de la misma es necesario incluir un grupo multidisciplinario que permita definir correctamente los objetivos del proceso que se desea trabajar y los parámetros de control que guíen el buen desarrollo del sistema lo cual permitirá garantizar la coherencia y fiabilidad de los resultados. Con respecto a este equipo, deberá estar conformado por las personas responsables del proceso que posean conocimiento y experiencia en el área de la decisión.

Una vez conformado el equipo, se procede con los pasos que a continuación se exponen. 


\subsection{Identificación de los qué o variables internas}

Se identifican los factores que las bodegas (el cliente interno) esperan que el centro de distribución (proveedor) cumpla en lo referente a la entrega del producto. Se debe obtener y expresar lo que en realidad quiere el cliente, y no lo que el proveedor "piensa" que el cliente espera [16]. En este caso es importante contactar directamente a los clientes y conocer sus requerimientos. Para ello, además del contacto directo, se podría utilizar una encuesta y a partir de los resultados consolidados definir esos requerimientos que serán considerados como los qué o las variables internas.

\subsection{Determinación de la importancia relativa o peso de los qué}

Cuando los clientes enuncien sus deseos (los qué) también deben expresar el nivel de importancia que tiene cada uno de ellos. Para lograrlo, se propone emplear una escala lingüística que se traducirá en números difusos triangulares para el manejo matemático respectivo. Un ejemplo de escala lingüística y su correspondiente representación en números difusos triangulares se presenta en la tabla $1 \mathrm{y}$ en la figura 1. La idea entonces es que, al mismo tiempo que se definan los criterios (qué), los clientes definan también la importancia de dichos criterios. A partir de esta calificación, se determina su importancia relativa a través de la ecuación (1):

$$
\begin{gathered}
\text { Pesos QUE }=\{\mathrm{Wi} \text {, donde } \mathrm{i}=1, \ldots \mathrm{K}\} \\
\mathrm{Wi}=\frac{1}{\mathrm{n}} *(\mathrm{Wi} 1+\mathrm{Wi} 2+\ldots+\mathrm{Win}) \\
\mathrm{Wi}=(\mathrm{Wi} \alpha, \mathrm{Wi} \beta, \mathrm{Wi} \gamma)
\end{gathered}
$$

Donde $K$ es el número de los qué y $n$ es el número de evaluadores, cada elemento del vector peso de los qué $\left(W_{i}\right)$, es un número triangular difuso. Los pesos finales se obtienen al sumar las calificaciones dadas por los evaluadores.

Tabla 1. Escala lingüística y números difusos triangulares equivalentes

\begin{tabular}{|l|c|}
\hline Variable lingüistica & Número difuso triangular $(a, b, c)$ \\
\hline Muy bajo (MB) & $(0,1,2)$ \\
\hline Bajo (B) & $(2,3,4)$ \\
\hline Medio (M) & $(4,5,6)$ \\
\hline Alto (A) & $(6,7,8)$ \\
\hline Muy alto (MA) & $(8,9,10)$ \\
\hline
\end{tabular}

Fuente: [7] 


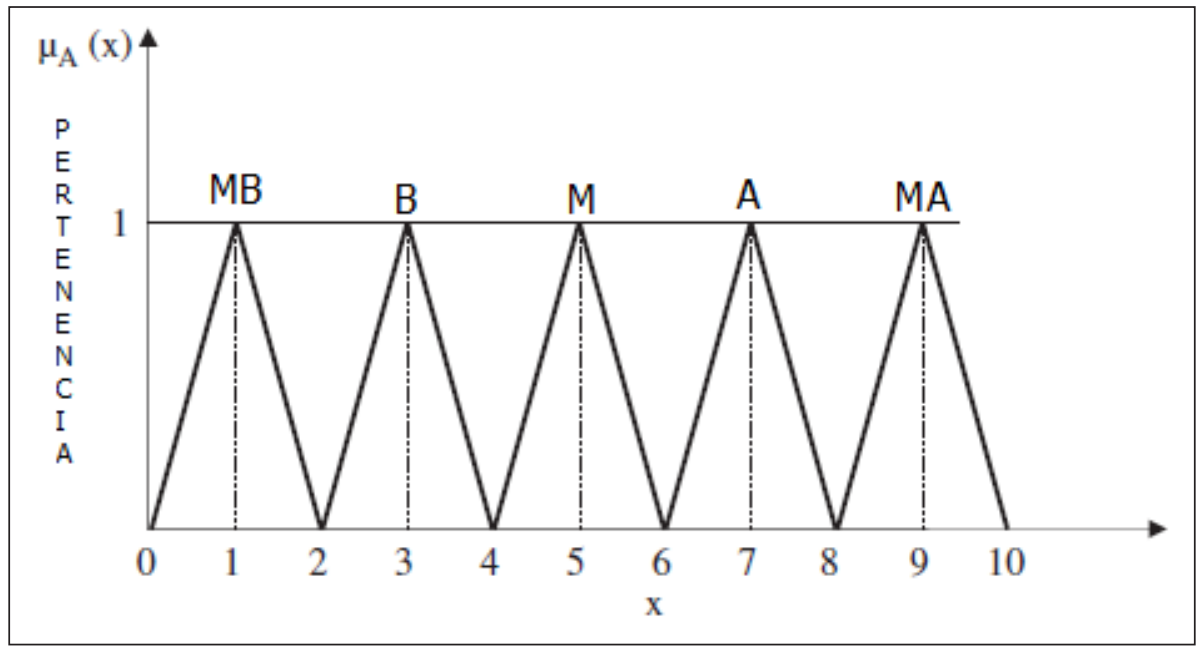

Figura 1. Escala cualitativa de calificación de importancia relativa Fuente: [2]

\subsection{Identificación de los cómo o variables externas}

Se identifican los criterios que, se espera, satisfagan las exigencias y necesidades expresadas por los clientes en el paso anterior. En este caso, los factores que respondan a dichas exigencias, deben ser criterios para el despacho de producto terminado. A continuación, se presentan los criterios que fueron considerados [17]:

- Criterio de cobertura en unidades (CU).

- Criterio de cobertura en días (CD).

- Criterio tamaño del faltante (TF).

- Criterio tamaño del pedido (TP).

- Criterio de demanda.

- Criterio de variabilidad de demanda.

- Priorización de zona de mercado.

- Costo logístico.

Es importante aclarar que podrían incluirse criterios adicionales de acuerdo con las particularidades empresariales, pero que se considera que los criterios aquí presentados tienen una cobertura amplia de la situación y que la abordan desde diferentes perspectivas, lo cual hace más completo el proceso de toma de decisión. 


\subsection{Determinación de los niveles de relación entre los qué y los cómo}

En este punto, cada miembro del equipo multidisciplinario expresa su opinión acerca del impacto que tiene cada variable externa (cómo) sobre cada variable interna (qué) por medio de la escala lingüística mostrada en la tabla 1. Después de que se han definido todas las correlaciones, se consolidan las diferentes opiniones para finalmente determinar el nivel de correlación entre las dos variables, utilizando la ecuación (2):

$$
\text { Correlación }=\{r i j, \text { donde } i=1, \ldots, k \text { y } j=\ldots \mathrm{m}\}
$$

$\mathrm{K}$ es el número de los qué, $\mathrm{m}$ el número de los cómo y $\mathrm{n}$ el número de miembros del equipo, aquí cada uno de los elementos rij representa el nivel de correlación consolidado entra cada $i$-qué y cada $j$-cómo. Estos se representan mediante números triangulares difusos de la forma que se aprecia en la ecuación (3):

$$
r i j=(r i j \propto, r i j \beta, r i j \gamma)
$$

\subsection{Cuantificación de la importancia relativa de cada cómo}

La importancia relativa de cada cómo se calcula promediando los valores de correlación rij consolidados con los valores de la importancia relativa de cada qué wj, a través de la ecuación (4):

$$
\begin{gathered}
\text { Pesos COMO }=\{W j, \text { donde } j=1, \ldots, \mathrm{m}\} \\
r i j=\frac{1}{\mathrm{n}} *(r i j 1+r i j 2+\ldots+r i j \mathrm{n}) \\
W j=\frac{1}{\mathrm{k}} *\left[\left(r j 1^{*} W 1\right)+\ldots+(r j \mathrm{k} * W \mathrm{k})\right]
\end{gathered}
$$

$\mathrm{K}$ es el número de los qué, $\mathrm{m}$ el número de los cómo, cada uno de los elementos $w j$, del vector peso-como representan la importancia relativa de cada cómo, la cual se define por medio de la función de números triangulares difusos representados en la ecuación (5):

$$
W j=(W j \propto, W j \beta, W j \gamma)
$$

\subsection{Cálculo del valor numérico de los cómo para cada bodega}

Se ingresa el valor numérico de cada cómo para cada una de las bodegas que se muestra en la tabla 5. En este paso, las variables externas (los cómo) se califican de manera cuantitativa obteniéndose los valores por medio de fórmulas matemáticas, asociados a los criterios presentados en el paso de la identificación de los cómo o variables externas y cuyas expresiones matemáticas se encuentran en [16]. 
Cada uno de estos criterios debe ser calculado antes de ser ingresados al modelo. Para los indicadores que no relacionan fórmulas matemáticas, se debe ingresar el dato numérico de la variable, por ejemplo, para el caso de la demanda, el valor de este indicador será la demanda mensual o semanal del producto expresada en unidades.

\subsection{Ranking final para despacho de producto terminado}

En este paso concluye el modelo QFD-difuso y se obtiene el orden final en el que se debe despachar el producto terminado. El ranking se calcula a través de un vector llamado pesos de los cómo, el cual se encuentra expresado en la ecuación (6):

$$
W j=(W j \propto, W j \beta, W j \gamma)
$$

Una vez obtenido este vector se utiliza el enfoque de clasificación de números difusos triangulares definido en [18], logrando de esta forma la calificación consolidada de la importancia relativa de cada cómo expresada en números naturales mediante la ecuación (7):

$$
C j=\frac{(W j \propto+2 * W j \beta+W j \gamma)}{4}
$$

Aplicando la ecuación (7) a cada vector, se obtiene una calificación de la forma $C 1, C 2, \ldots, C m$ para cada uno.

Al contar con la calificación de las variables externas de la forma $C j$ se realiza la sumatoria de las mismas y así se obtiene una ponderación de cada criterio $(P j)$; para esto se utiliza la ecuación (8):

$$
Z=\sum c_{j} \quad P_{j}=-
$$

Donde:

$$
\underline{\mathrm{Cb}}
$$

Se obtiene una ponderación $P j$ de cada variable externa de la forma $P 1, P 2, \ldots, P n$.

Finalmente, multiplica los $P j$ por el valor de la bodega respecto a la misma variable externa calificada de forma cuantitativa para cada una de estas, de acuerdo con el paso del cálculo del valor numérico de los cómo para cada bodega.

$C j$ : calificación consolidada de cada cómo.

$P j$ : ponderación cualitativa de cada cómo. 
El puntaje final se obtiene al sumar los valores obtenidos de las multiplicaciones anteriores para cada bodega. Respecto a este puntaje se establece el ranking en orden de mayor a menor siendo la bodega del puntaje más alto, la primera a abastecer, continuando así hasta el abastecimiento de la bodega de menor puntaje. A continuación, se presenta un ejemplo en el cual se ilustra la aplicación de esta metodología propuesta.

\section{RESULTADOS. EJEMPLO DE APLICACIÓN}

Para el caso de estudio que se presenta a continuación se obtuvo información de empresas manufactureras de la ciudad de Cali y sus zonas aledañas.

\subsection{Identificar los qué o variables internas}

Son los factores que las bodegas esperan que el proveedor cumpla en cuanto a la distribución y entrega de producto. Estos se convierten en las variables internas (los qué).

- Cumplimiento de hora y fechas de entrega.

- Calidad de las mercancías.

- Pedidos completos.

- Respuesta oportuna a novedades, inconvenientes y reclamos.

- Cumplimiento con la documentación requerida.

- Seguridad del producto.

\subsection{Identificar los criterios relevantes para la evaluación de los proveedores. Los cómo o variables externas}

Los criterios que satisfacen las exigencias y necesidades expresadas por el cliente fueron propuestos como las variables externas:

- Criterio de cobertura en unidades (CU).

- Criterio de cobertura en días (CD).

- Criterio tamaño del faltante (TF).

- Criterio tamaño del pedido (TP).

- Criterio de demanda.

- Criterio de variabilidad de demanda.

- Priorización de zona de mercado.

- Costo logístico. 


\subsection{Determinar la importancia relativa de las variables internas}

En este caso, se trabajó con la escala lingüística presentada en la tabla 1. Estas calificaciones de carácter cualitativo, se cuantifican mediante la lógica difusa a través de los números triangulares difusos presentados en la tabla 1 e ilustrados en la figura 1. Para la aplicación práctica, la tabla 2 muestra las opiniones de los tres involucrados.

Tabla 2. Calificación de los qué

\begin{tabular}{|c|c|c|c|c|}
\hline & \multirow{3}{*}{$\frac{\text { Jefe de logística }}{\text { MA }}$} & \multirow{3}{*}{$\begin{array}{c}\text { Supervisor de } \\
\text { bodega }\end{array}$} & \multirow{3}{*}{$\frac{\text { Jefe de bodega }}{\text { A }}$} \\
\hline & & & & \\
\hline \multirow{6}{*}{ 近 } & Cumplimiento de hora y fecha de entrega & & & \\
\hline & Calidad de las mercancías & MA & A & MA \\
\hline & Pedidos completos & A & M & M \\
\hline & $\begin{array}{l}\text { Respuesta oportuna a novedades, } \\
\text { inconvenientes y reclamos }\end{array}$ & A & MA & $\mathrm{M}$ \\
\hline & $\begin{array}{l}\text { Cumplimiento con la documentación } \\
\text { requerida }\end{array}$ & $\mathrm{B}$ & MA & M \\
\hline & Seguridad del producto & M & A & $\mathrm{A}$ \\
\hline
\end{tabular}

Fuente: elaboración propia

\subsection{Cuantificar la importancia relativa de cada variable externa}

La tabla 3 muestra la calificación dada por cada uno de los involucrados para el nivel de correlación que existe entre cada variable externa y cada variable interna en el caso de estudio.

Tabla 3. Correlación entre los qué y los cómo

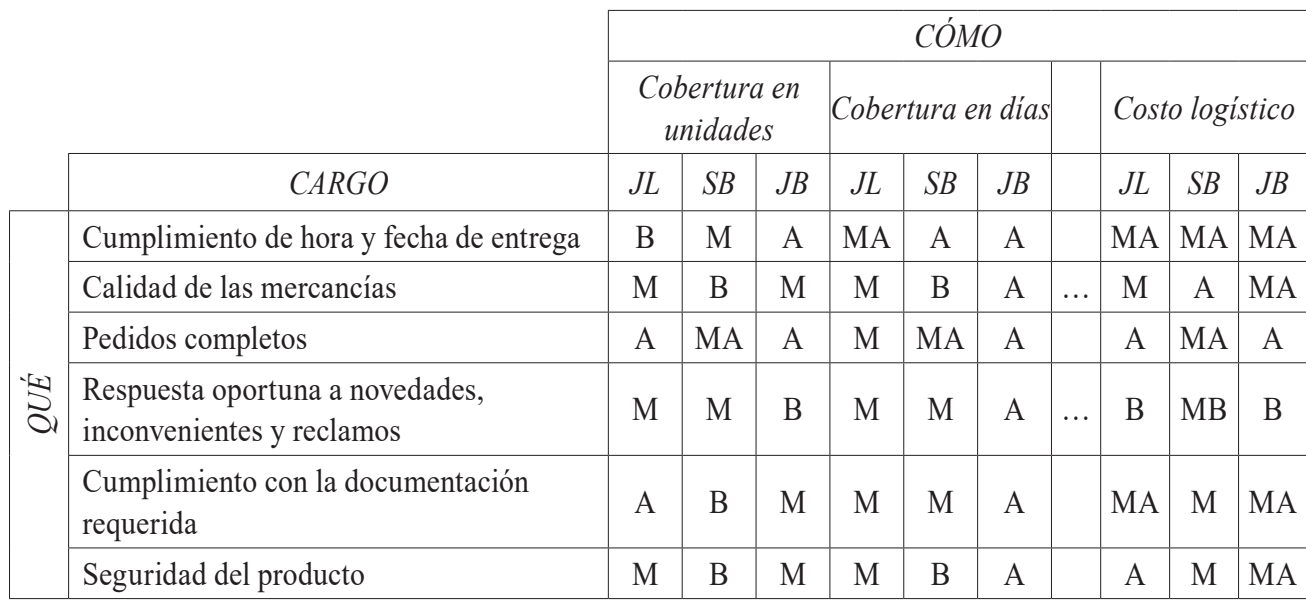

Fuente: elaboración propia 


\subsection{Determinar los niveles de correlación entre las variables internas y externas Continuando con la metodología, se definieron los niveles de correlación para las variables tanto internas como externas tal como se presentan en la tabla 4.}

Tabla 4. Correlación entre las variables internas y externas

\begin{tabular}{|c|c|c|c|c|c|c|c|c|c|c|c|c|c|}
\hline \multirow[b]{2}{*}{ Cumplimiento de hora y fecha de entrega } & \multicolumn{3}{|c|}{$\begin{array}{l}\text { Peso de los } \\
\text { qué }\end{array}$} & \multicolumn{3}{|c|}{$\begin{array}{l}\text { Cobertura en } \\
\text { unidades }\end{array}$} & \multicolumn{3}{|c|}{$\begin{array}{c}\text { Cobertura en } \\
\text { dias }\end{array}$} & & \multicolumn{3}{|c|}{$\begin{array}{c}\text { Costo } \\
\text { logístico }\end{array}$} \\
\hline & 7 & 8 & 9 & 4 & 5 & 6 & 6,7 & 7,7 & 8,7 & & 8 & 9 & 10 \\
\hline Calidad de las mercancías & 7 & 8 & 9 & 3,3 & 4,3 & 5,3 & 4 & 5 & 6 & $\ldots$ & 6 & 7 & 8 \\
\hline Pedidos completos & 5 & 6 & 7 & 6,7 & 7,7 & 8,7 & 6 & 7 & 8 & & 6,7 & 7,7 & 8,7 \\
\hline $\begin{array}{l}\text { Respuesta oportuna a novedades, inconve- } \\
\text { nientes y reclamos }\end{array}$ & 6 & 7 & 8 & 3,3 & 4,3 & 5,3 & 4,7 & 5,7 & 6,7 & .. & 1,3 & 2,3 & 3,3 \\
\hline $\begin{array}{l}\text { Cumplimiento con la documentación re- } \\
\text { querida }\end{array}$ & 5 & 6 & 7 & 4 & 5 & 6 & 4,7 & 5,7 & 6,7 & & 6,7 & 7,7 & 8,7 \\
\hline Seguridad del producto & 5 & 6 & 7 & 3,3 , & 4,3 & 5,3 & 4 & 5 & 6 & & 6 & 7 & 8 \\
\hline & & & & \multicolumn{10}{|c|}{ Peso de los cómo } \\
\hline & & & & 24 & 35 & 48 & 30 & 41 & 55 & $\ldots$ & 34 & 47 & 61 \\
\hline
\end{tabular}

Fuente: elaboración propia

\subsection{Determinar el impacto final de cada bodega sobre los atributos considerados Los resultados obtenidos se presentan en la tabla 5 .}

Tabla 5. Valores de los indicadores de cada bodega

\begin{tabular}{|c|c|c|c|c|c|}
\hline & \multicolumn{4}{|c|}{ CÓMOS } \\
\hline & $B O D E G A S$ & Cobertura en unidades & Cobertura en dias & & Costo logístico \\
\hline \multirow{10}{*}{ 式 } & Bodega A & -223 & -1 & & 2500 \\
\hline & Bodega B & 228 & 1 & & 3000 \\
\hline & Bodega C & 533 & 2 & $\ldots$ & 1500 \\
\hline & Bodega D & -123 & 0 & & 450 \\
\hline & Bodega $\mathrm{E}$ & 360 & 1 & & 1860 \\
\hline & Bodega $\mathrm{F}$ & 12 & 0 & & 1200 \\
\hline & Bodega G & 144 & 1 & $\ldots$ & 50 \\
\hline & Bodega $\mathrm{H}$ & -67 & 0 & & 2450 \\
\hline & Bodega I & 261 & 1 & & 1350 \\
\hline & Bodega J & 134 & 0 & & 145 \\
\hline
\end{tabular}

Fuente: elaboración propia 


\subsection{Clasificación final de las alternativas (ranking de las bodegas)}

En este caso particular, el ranking establecido se muestra en la tabla 6. Este ranking define la prioridad de despachos, lo cual quiere decir que las bodegas deberían ser atendidas en el orden presentado y en el evento que se agoten los recursos (camiones, o la misma producción), habrán sido abastecidas las bodegas prioritarias.

Tabla 6. Ranking de las bodegas

\begin{tabular}{|l|l|}
\hline \multicolumn{2}{|c|}{ Ranking } \\
\hline Bodega A & 433 \\
\hline Bodega B & 417 \\
\hline Bodega C & 410 \\
\hline Bodega D & 391 \\
\hline Bodega E & 372 \\
\hline Bodega F & 347 \\
\hline Bodega G & 317 \\
\hline Bodega H & 289 \\
\hline Bodega I & 276 \\
\hline Bodega J & 257 \\
\hline
\end{tabular}

Fuente: elaboración propia

Como los pesos ya fueron definidos, al actualizar los valores de los indicadores en cada bodega, producto del abastecimiento, las prioridades cambiarán y el proceso se repetirá de manera que todas las bodegas serán atendidas a medida que avanza el tiempo. El proceso es dinámico y en el evento que se definan otros indicadores, puede realizarse nuevamente la valoración por parte de los expertos de acuerdo con la metodología presentada.

\section{CONCLUSIONES}

El modelo presentado es de fácil adaptación a otras circunstancias o situaciones: los nuevos requisitos de las bodegas pueden ser fácilmente adaptadas al modelo, al igual que los cómo con los que se busca satisfacer estos nuevos requisitos. En cualquier análisis de sensibilidad que se realice, se evidenciará la influencia de las variables lingüísticas y cuantitativas en el orden de despacho resultante. Al suponer cambios en las opiniones subjetivas respecto a los cómo del modelo, se podrá observar que este no trata de forma aislada las variables cualitativas y cuantitativas sino que, por el contrario, ambos tipos de variables son tenidos en cuenta de forma simultánea para llegar a un orden de despacho. 
La forma en que se relacionan las variables lingüísticas y cuantitativas en este modelo hace que las decisiones a las que se llegan sean decisiones acordes a la luz de los indicadores logísticos, al tiempo que incorporan a dichas decisiones la opinión de las personas expertas en el tema.

La herramienta QFD-difuso permite incorporar al modelo indicadores logísticos, calculados por medio de fórmulas matemáticas. De igual manera, mediante QFDdifuso se hace posible incorporar al modelo QFD las opiniones subjetivas de las personas involucradas en la toma de decisiones por medio de la lógica difusa. Con lo que el modelo en su totalidad permite tomar una decisión integral, orientada hacia la satisfacción de todos los criterios de manera global.

\section{REFERENCIAS}

[1] J.J. Bravo, J.C. Osorio y J.P. Orejuela, "Modelo para la priorización dinámica de despachos de vehículos utilizando el proceso analítico jerárquico," Revista Facultad de Ingeniería, N. 48, 201- 215, 2009.

[2] L-K. Chan y M-L. Wu, “Quality function deployment: A literature review," European Journal of Operational Research, vol. 143, N. ${ }^{\circ} 3,463-497,2002$

[3] J. A. Carnevalli y P. Cauchick P., "Review, analysis and classification of the literature on QFD-Types of research, difficulties and benefits," International Journal of Production Economics, vol. 114, N. ${ }^{\circ} 2,737-754,2008$.

[4] M-T. Tsai, H-L. Wu y W-K. Liang, "Fuzzy Decision Making for Market Positioning and Developing Strategy for Improving Service Quality in Department Stores," Quality and Quantity, vol. 42, N.`3, $303-319,2008$.

[5] L. Lazzari, E. Machado y R. Pérez, Teoría de decisión fuzzy, Buenos Aires: Ed. Macchi, 1998.

[6] E. Bottani y A. Rizzi, "Strategic management of logistics service: A fuzzy QFD approach," International Journal of Production Economics, vol. 103, N. ${ }^{\circ}$ 2, 585-599, 2006.

[7] E. Bottani, "A fuzzy QFD approach to achieve agility," International Journal of Production Economics, vol. 119, N. ${ }^{\circ}$ 2, 380-391, 2009.

[8] M. Celik, C.Selcuk, C. Kahraman y E.Deha, "An integrated fuzzy QFD model proposal on routing of shipping investment decisions in crude oil tanker market," Expert Systems with Applications, vol 36, N. ${ }^{\circ} 3,6227-6235,2009$.

[9] Y, Sohn So y I.S. Choi, "Fuzzy QFD for supply chain management with reliability consideration," Reliability Engineering and System Safety, vol. 22, N. ${ }^{\circ}$ 3, 327-334, 2001.

[10] L-H. Chen y W-C. Ko, "A fuzzy nonlinear model for quality function deployment considering Kano's concept," Mathematical and Computer Modelling, vol. 48, N. ${ }^{\circ}$ 3, 581-593, 2008. 
[11] H-T. Liu, "The extension of fuzzy QFD: From product planning to part deployment," Expert Systems with Applications, vol. 36, N. . 8, 11131-11144, 2009.

[12] S. H. Amin y J. Razmi, "An integrated fuzzy model for supplier management: A case study of ISP selection and evaluation," Expert Systems with Applications, vol. 36, N. 4, 8639-8648, 2009.

[13] D. C. Arango y C.E. Ruales, "Selección de proveedores mediante el despliegue de la función de calidad difuso," Trabajo de grado de pregrado, Universidad del Valle, Cali, 2008.

[14] D. J. Arias y M. A. Peña, "Formulación de un modelo para priorización de despacho de producto terminado en empresas manufactureras usando QFD fuzzy," Trabajo de grado de pregrado, Universidad del Valle, Cali, 2010.

[15] Giraldo P y Martínez L (2009). "Propuesta de mejoramiento para el programa de ingeniería industrial de la universidad del valle utilizando QFD-fuzzy," Trabajo de grado de pregrado, Universidad del Valle, Cali, 2009.

[16] M.F. Herrera y J.C. Osorio, "Modelo para la gestión de proveedores utilizando AHP difuso," Estudios Gerenciales Universidad ICESI, N. 99, pp. 69-88, 2006.

[17] J.J. Bravo, J.P. Orejuela y J.C. Osorio, “Administración de recursos de distribución: Indicadores para la priorización en transporte," Estudios Gerenciales Universidad ICESI, vol 33, N. 102 , pp. 101-118, 2007.

[18] G. Facchinetti, "Ranking functions induced by weighted average of fuzzy numbers," Fuzzy Sets and Systems, vol. 1, pp. 313-327, 2002. 